\title{
FIXED POINT ITERATION PROCESSES FOR ASYMPTOTICALLY NONEXPANSIVE MAPPINGS
}

\author{
KOK-KEONG TAN AND HONG-KUN XU
}

(Communicated by Palle E. T. Jorgensen)

\begin{abstract}
Let $X$ be a uniformly convex Banach space which satisfies Opial's condition or has a Fréchet differentiable norm, $C$ a bounded closed convex subset of $X$, and $T: C \rightarrow C$ an asymptotically nonexpansive mapping. It is then shown that the modified Mann and Ishikawa iteration processes defined by $x_{n+1}=t_{n} T^{n} x_{n}+\left(1-t_{n}\right) x_{n}$ and $x_{n+1}=t_{n} T^{n}\left(s_{n} T^{n} x_{n}+\left(1-s_{n}\right) x_{n}\right)+\left(1-t_{n}\right) x_{n}$, respectively, converge weakly to a fixed point of $T$.
\end{abstract}

\section{INTRODUCTION}

Let $C$ be a nonempty subset of a Banach space $X$. A mapping $T: C \rightarrow C$ is said to be asymptotically nonexpansive if there exists a sequence $\left\{k_{n}\right\}$ of positive numbers with $\lim _{n \rightarrow \infty} k_{n}=1$ such that

$$
\left\|T^{n} x-T^{n} y\right\| \leq k_{n}\|x-y\|
$$

for all $x, y$ in $C$ and $n=1,2, \ldots$. This class of mappings, as a natural extension to that of nonexpansive mappings, was introduced by Goebel and Kirk [4] in 1972. They proved that if $C$ is a bounded closed convex subset of a uniformly convex Banach space $X$, then every asymptotically nonexpansive self-mapping $T$ of $C$ has a fixed point. This existence result was recently generalized in [14] to a nearly uniformly convex (NUC) Banach space setting (see [5] for definition).

The study of iterative construction for fixed points of asymptotically nonexpansive mappings began in 1978. Bose [1] first proved that if $C$ is a bounded closed convex subset of a uniformly convex Banach space $X$ which satisfies Opial's condition [7] and if $T: C \rightarrow C$ is an asymptotically nonexpansive mapping, then $\left\{T^{n} x\right\}$ converges weakly to a fixed point of $T$ provided $T$ is asymptotically regular at $x$, i.e., $\lim _{n \rightarrow \infty}\left\|T^{n} x-T^{n+1} x\right\|=0$. This conclusion is still valid $[8,14]$ if Opial's condition of $X$ is replaced by the condition that $X$ has a Fréchet differentiable norm. Furthermore, in both cases, asymptotic regularity of $T$ at $x$ can be weakened to weak asymptotic regularity of $T$ at $x$, i.e., $\mathrm{w}-\lim _{n \rightarrow \infty}\left(T^{n} x-T^{n+1} x\right)=0$ (see $\left.[12,13]\right)$.

Received by the editors January 21, 1992 and, in revised form, February 9, 1993.

1991 Mathematics Subject Classification. Primary 47H09, 47H10; Secondary 46B20.

Key words and phrases. Fixed point, asymptotically nonexpansive mapping, fixed point iteration process, uniformly convex Banach space, Fréchet differentiable norm, Opial's condition. 
Recently, Schu [10] considered the following modified Mann iteration process:

$$
x_{n+1}=t_{n} T^{n} x_{n}+\left(1-t_{n}\right) x_{n}, \quad n \geq 1,
$$

where $\left\{t_{n}\right\}$ is a sequence of real numbers in $(0,1)$ which is bounded away from both 0 and 1, i.e., $a \leq t_{n} \leq b$ for all $n$ and some $0<a \leq b<1$. He verified that if $C$ is a bounded closed convex subset of a Banach space $X$ satisfying Opial's condition and if $T: C \rightarrow C$ is an asymptotically nonexpansive mapping such that $\sum_{n=1}^{\infty}\left(k_{n}-1\right)$ converges, then the modified Mann iteration process (M) converges weakly to a fixed point of $T$. Unfortunately, Schu's theorem does not apply to the $L^{p}$ spaces if $p \neq 2$ since none of these spaces satisfy Opial's condition (cf. [7]).

In this paper we first show that Schu's theorem remains true if the assumption that $X$ satisfies Opial's condition is replaced by the one that $Y$ has a Fréchet differentiable norm. This result (Theorem 3.1) applies to the $L^{p}$ spaces for $1<p<\infty$ since each of these spaces is uniformly convex and uniformly smooth. We then prove the weak convergence of the modified Ishikawa iteration process (cf. Ishikawa [6]):

$$
x_{n+1}=t_{n} T^{n}\left(s_{n} T^{n} x_{n}+\left(1-s_{n}\right) x_{n}\right)+\left(1-t_{n}\right) x_{n}, \quad n \geq 1,
$$

in a uniformly convex Banach space which either satisfies Opial's condition or has a Fréchet differentiable norm.

\section{Preliminaries AND lemmas}

Let $X$ be a Banach space. Recall that $X$ is said to satisfy Opial's condition [7] if for each sequence $\left\{x_{n}\right\}$ in $X$ the condition $x_{n} \rightarrow x$ weakly implies $\overline{\lim }_{n \rightarrow \infty}\left\|x_{n}-x\right\|<\overline{\lim }_{n \rightarrow \infty}\left\|x_{n}-y\right\|$ for all $y \in X$ different from $x$. It is known [7] that each $l^{p} \quad(l \leq p<\infty)$ enjoys this property, while $L^{p}$ does not unless $p=2$. It is also known [3] that any separable Banach space can be equivalently renormed so that it satisfies Opial's condition. Recall also that $X$ is said to have a Fréchet differentiable norm if, for each $x$ in $S(X)$, the unit sphere of $X$, the limit

$$
\lim _{t \rightarrow 0} \frac{\|x+t y\|-\|x\|}{t}
$$

exists and is attained uniformly in $y \in S(X)$. In this case, we have

$$
\frac{1}{2}\|x\|^{2}+\langle h, J(x)\rangle \leq \frac{1}{2}\|x+h\|^{2} \leq \frac{1}{2}\|x\|^{2}+\langle h, J(x)\rangle+b(\|h\|)
$$

for all $x, h \in X$, where $J$ is the normalized duality map from $X$ to $X^{*}$ defined by

$$
J(x)=\left\{x^{*} \in X^{*}:\left\langle x, x^{*}\right\rangle=\|x\|^{2}=\left\|x^{*}\right\|^{2}\right\},
$$

$\langle\cdot, \cdot\rangle$ is the duality pairing between $X$ and $X^{*}$, and $b$ is a function defined on $[0, \infty)$ such that $\lim _{t \downarrow 0} b(t) / t=0$.

Suppose now that $C$ is a bounded closed convex subset of a Banach space $X$ and $\left\{T_{n}\right\}$ is a sequence of Lipschitzian self-mappings of $C$ such that the set $F$ of common fixed points of $\left\{T_{n}\right\}$ is nonempty. Denote by $L_{n}$ the Lipschitz constant of $T_{n}$. In the sequel, we always assume $L_{n} \geq 1$ for all $n \geq 1$ and use the notations $\overline{\mathrm{lim}}=\lim \sup , \underline{\lim }=\lim$ inf, $\rightarrow$ for weak convergence, $\rightarrow$ for strong convergence, and $F(T)$ for the set of fixed points of $T$. 
For a given $x_{1} \in C$, we recurrently define the sequence $\left\{x_{n}\right\}$ by

$$
x_{n+1}=T_{n} x_{n}, \quad n \geq 1 .
$$

Lemma 2.1. Suppose that $\sum_{n}\left(L_{n}-1\right)$ converges. Then for each $f \in F$, $\lim _{n}\left\|x_{n}-f\right\|$ exists.

Proof. For all $n, m \geq 1$, we have

$$
\begin{aligned}
\left\|x_{n+m+1}-f\right\| & =\left\|T_{n+m} x_{n+m}-f\right\| \leq L_{n+m}\left\|x_{n+m}-f\right\| \\
& \leq\left(\prod_{j=n}^{n+m} L_{j}\right)\left\|x_{n}-f\right\| .
\end{aligned}
$$

Since $\sum_{n}\left(L_{n}-1\right)$ converges, it follows that

$$
\varlimsup_{m \rightarrow \infty}\left\|x_{n+m+1}-f\right\| \leq\left(\prod_{j=n}^{\infty} L_{j}\right)\left\|x_{n}-f\right\| .
$$

Consequently,

$$
\varlimsup_{n}\left\|x_{n}-f\right\| \leq \frac{\lim }{n}\left\|x_{n}-f\right\| .
$$

This proves the lemma.

Lemma 2.2. Suppose that $X$ is uniformly convex and $\sum_{n}\left(L_{n}-1\right)$ converges. Then $\lim _{n \rightarrow \infty}\left\|t x_{n}+(1-t) f_{1}-f_{2}\right\|$ exists for every $f_{1}, f_{2} \in F$ and $0 \leq t \leq 1$. Proof. We follow an idea of Reich [9]. Set

$$
a_{n}=a_{n}(t)=\left\|t x_{n}+(1-t) f_{1}-f_{2}\right\|, \quad S_{n, m}=T_{n+m-1} T_{n+m-2} \cdots T_{n},
$$

and

$$
b_{n, m}=\left\|S_{n, m}\left(t x_{n}+(1-t) f_{1}\right)-\left(t x_{n+m}+(1-t) f_{1}\right)\right\| .
$$

Then, observing $S_{n, m} x_{n}=x_{n+m}$, we get

$$
\begin{aligned}
a_{n+m} & =\left\|t x_{n+m}+(1-t) f_{1}-f_{2}\right\| \\
& \leq b_{n, m}+\left\|S_{n, m}\left(t x_{n}+(1-t) f_{1}\right)-f_{2}\right\| \\
& \leq b_{n, m}+\left(\prod_{j=n}^{n+m-1} L_{j}\right) a_{n} \leq b_{n, m}+H_{n} a_{n},
\end{aligned}
$$

where $H_{n}=\prod_{j=n}^{\infty} L_{j}$. By a result of Bruck [2], we have

$$
\begin{aligned}
b_{n, m} & \leq H_{n} g^{-1}\left(\left\|x_{n}-f_{1}\right\|-H_{n}^{-1}\left\|S_{n, m} x_{n}-f_{1}\right\|\right) \\
& \leq H_{n} g^{-1}\left(\left\|x_{n}-f_{1}\right\|-\left\|x_{n+m}-f_{1}\right\|+\left(1-H_{n}^{-1}\right) d\right),
\end{aligned}
$$

where $g:[0, \infty) \rightarrow[0, \infty), g(0)=0$, is a strictly increasing continuous function depending only on $d$, the diameter of $C$. Since $\lim _{n \rightarrow \infty} H_{n}=1$, it follows from Lemma 2.1 that $\lim _{n, m \rightarrow \infty} b_{n, m}=0$. Therefore,

$$
\varlimsup_{m \rightarrow \infty} a_{m} \leq \lim _{n, m \leq \infty} b_{n, m}+\underline{\lim }_{n \rightarrow \infty} H_{n} a_{n}=\varliminf_{n \rightarrow \infty} a_{n} .
$$

This completes the proof. 
Lemma 2.3. Suppose that $X$ is a uniformly convex Banach space with a Fréchet differentiable norm and that $\sum_{n}\left(L_{n}-1\right)$ converges. Then for every $f_{1}, f_{2} \in F$, $\lim _{n \rightarrow \infty}\left\langle x_{n}, J\left(f_{1}-f_{2}\right)\right\rangle$ exists; in particular,

$$
\left\langle p-q, J\left(f_{1}-f_{2}\right)\right\rangle=0
$$

for all $p, q \in \omega_{w}\left(x_{n}\right)$. Here, $\omega_{w}\left(x_{n}\right)$ denotes the weak $\omega$-limit set of $\left\{x_{n}\right\}$, i.e., $\omega_{w}\left(x_{n}\right)=\left\{y \in X: y=\mathrm{w}-\lim _{k \rightarrow \infty} x_{n_{k}}\right.$ for some $\left.n_{k} \uparrow \infty\right\}$.

Proof. Taking $x=f_{1}-f_{2}$ and $h=t\left(x_{n}-f_{1}\right)$ in (2.1), we get

$$
\begin{gathered}
\frac{1}{2}\left\|f_{1}-f_{2}\right\|^{2}+t\left\langle x_{n}-f_{1}, J\left(f_{1}-f_{2}\right)\right\rangle \leq \frac{1}{2}\left\|t x_{n}+(1-t) f_{1}-f_{2}\right\|^{2} \\
\quad \leq \frac{1}{2}\left\|f_{1}-f_{2}\right\|^{2}+t\left\langle x_{n}-f_{1}, J\left(f_{1}-f_{2}\right)\right\rangle+b\left(t\left\|x_{n}-f_{1}\right\|\right) .
\end{gathered}
$$

It follows from Lemma 2.2 that

$$
\begin{aligned}
\frac{1}{2} \| f_{1} & -f_{2} \|^{2}+t \cdot \varlimsup_{n \rightarrow \infty}\left\langle x_{n}-f_{1}, J\left(f_{1}-f_{2}\right)\right\rangle \\
& \leq \lim _{n \rightarrow \infty} \frac{1}{2}\left\|t x_{n}+(1-t) f_{1}-f_{2}\right\|^{2} \\
& \leq \frac{1}{2}\left\|f_{1}-f_{2}\right\|^{2}+t \cdot \underline{\lim }_{n \rightarrow \infty}\left\langle x_{n}-f_{1}, J\left(f_{1}-f_{2}\right)\right\rangle+o(t) .
\end{aligned}
$$

This yields

$$
\varlimsup_{n \rightarrow \infty}\left\langle x_{n}-f_{1}, J\left(f_{1}-f_{2}\right)\right\rangle \leq \varliminf_{n \rightarrow \infty}\left\langle x_{n}-f_{1}, J\left(f_{1}-f_{2}\right)\right\rangle+o(1) .
$$

Letting $t \rightarrow 0^{+}$, we see that $\lim _{n \rightarrow \infty}\left\langle x_{n}-f_{1}, J\left(f_{1}-f_{2}\right)\right\rangle$ exists.

We also need the following known lemmas.

Lemma 2.4 (cf. Schu [10]). Let $X$ be a uniformly convex Banach space, $\left\{t_{n}\right\}$ a sequence of real numbers in $(0,1)$ bounded away from 0 and 1 , and $\left\{x_{n}\right\}$ and $\left\{y_{n}\right\}$ sequences of $X$ such that $\varlimsup_{n \rightarrow \infty}\left\|x_{n}\right\| \leq a, \varlimsup_{n \rightarrow \infty}\left\|y_{n}\right\| \leq a$, and $\lim _{n \rightarrow \infty}\left\|t_{n} x_{n}+\left(1-t_{n}\right) y_{n}\right\|=a$ for some $a \geq 0$. Then $\lim _{n \rightarrow \infty}\left\|x_{n}-y_{n}\right\|=0$.

Lemma 2.5 [11]. Let $X$ be a normed space, $C$ a convex subset of $X$, and $T: C \rightarrow C$ a uniformly L-Lipschitzian mapping, i.e., $\left\|T^{n} x-T^{n} y\right\| \leq L\|x-y\|$ for all $x, y$ in $C$ and $n=1,2, \ldots$ For any given $x_{1}$ in $C$ and sequences $\left\{t_{n}\right\}$ and $\left\{s_{n}\right\}$ in $[0,1]$, define $\left\{x_{n}\right\}$ by

$$
x_{n+1}=t_{n} T^{n}\left(s_{n} T^{n} x_{n}+\left(1-s_{n}\right) x_{n}\right)+\left(1-t_{n}\right) x_{n}, \quad n \geq 1 .
$$

Then we have

$$
\left\|x_{n}-T x_{n}\right\| \leq c_{n}+c_{n-1} L(1+3 L+2 L)^{2}
$$

for all $n \geq 2$, where $c_{n}=\left\|x_{n}-T^{n} x_{n}\right\|$.

Lemma 2.6 [14]. Suppose that $C$ is a bounded closed convex subset of a uniformly convex Banach space and $T: C \rightarrow C$ is an asymptotically nonexpansive mapping. Then $I-T$ is demiclosed at the origin, i.e., for any sequence $\left\{x_{n}\right\}$ in $C$, the conditions $x_{n}-x_{0}$ and $x_{n}-T x_{n} \rightarrow 0$ imply $x_{0}-T x_{0}=0$.

\section{WEAK CONVERGENCE}

In this section we prove the weak convergence of the modified Mann and the modified Ishikawa iteration processes in a uniformly convex Banach space which satisfies Opial's condition or has a Fréchet differentiable norm. 
Theorem 3.1. Let $X$ be a uniformly convex Banach space with a Fréchet differentiable norm, $C$ a bounded closed convex subset of $X$, and $T: C \rightarrow C$ an asymptotically nonexpansive mapping such that $\sum_{n}\left(k_{n}-1\right)$ converges. Then for each $x_{1} \in C$, the sequence $\left\{x_{n}\right\}$ defined by the modified Mann iteration process (M) with $\left\{t_{n}\right\}$ a sequence of real numbers bounded away from 0 and 1 converges weakly to a fixed point of $T$.

Proof. Set $T_{n}=t_{n} T^{n}+\left(1-t_{n}\right) I$. (Here $I$ is the identity operator of $X$.) Then it is easily seen that $x_{n+1}=T_{n} x_{n}, F\left(T_{n}\right) \supseteq F(T)$, and $T_{n}$ is Lipschitzian with constant $L_{n}=t_{n} k_{n}+\left(1-t_{n}\right) \geq 1$. Since $L_{n}-1=t_{n}\left(k_{n}-1\right) \leq k_{n}-1$ and $\sum_{n}\left(k_{n}-1\right)$ converges, $\sum_{n}\left(L_{n}-1\right)$ also converges. It thus follows from Lemma 2.3 that

$$
\left\langle p-q, J\left(f_{1}-f_{2}\right)\right\rangle=0
$$

for all $p, q \in \omega_{w}\left(x_{n}\right)$ and $f_{1}, f_{2} \in F(T)$. Moreover, for $f \in F(T)$, we have

$$
\varlimsup_{n \rightarrow \infty}\left\|T^{n} x_{n}-f\right\| \leq \varlimsup_{n \rightarrow \infty} k_{n}\left\|x_{n}-f\right\|=\lim _{n \rightarrow \infty}\left\|x_{n}-f\right\|
$$

and

$$
\lim _{n \rightarrow \infty}\left\|t_{n}\left(T^{n} x_{n}-f\right)+\left(1-t_{n}\right)\left(x_{n}-f\right)\right\|=\lim _{n \rightarrow \infty}\left\|x_{n+1}-f\right\| .
$$

It follows from Lemma 2.4 that $\lim _{n \rightarrow \infty}\left\|T^{n} x_{n}-x_{n}\right\|=0$, which implies by Lemma 2.5 that $\lim _{n \rightarrow \infty}\left\|x_{n}-T x_{n}\right\|=0$, which in turn implies by Lemma 2.6 that $\omega_{w}\left(x_{n}\right)$ is contained in $F(T)$. So to show that $\left\{x_{n}\right\}$ converges weakly to a fixed point of $T$, it suffices to show that $\omega_{w}\left(x_{n}\right)$ consists of just one point. To this end, let $p, q$ be in $\omega_{w}\left(x_{n}\right)$. Then since $p, q$ belong to $F(T)$, it follows from (3.1) that

$$
\|p-q\|^{2}=\langle p-q, J(p-q)\rangle=0 .
$$

Therefore, $p=q$ and the proof is complete.

Remark. We do not know whether Theorem 3.1 remains valid if $k_{n}$ is allowed to approach 1 slowly enough so that $\sum_{n}\left(k_{n}-1\right)$ diverges.

Next, we consider the modified Ishikawa iteration process (I) described in $\S 1$.

Theorem 3.2. Let $X$ be a uniformly convex Banach space which satisfies Opial's condition or has a Fréchet differentiable norm, $C$ a bounded closed convex subset of $X$, and $T: C \rightarrow C$ an asymptotically nonexpansive mapping such that $\sum_{n}\left(k_{n}-1\right)$ converges. Suppose that $x_{1}$ is a given point in $C$ and $\left\{t_{n}\right\}$ and $\left\{s_{n}\right\}$ are real sequences such that $\left\{t_{n}\right\}$ is bounded away from 0 and 1 and $\left\{s_{n}\right\}$ is bounded away from 1 . Then the sequence $\left\{x_{n}\right\}$ defined by the modified Ishikawa iteration process (I) converges weakly to a fixed point of $T$.

Proof. Define a mapping $T_{n}: C \rightarrow C$ by

$$
T_{n} x=t_{n} T^{n}\left(s_{n} T^{n} x+\left(1-s_{n}\right) x\right)+\left(1-t_{n}\right) x, \quad x \in C .
$$

Then it is easily seen that $x_{n+1}=T_{n} x_{n}, F\left(T_{n}\right) \supseteq F(T)$, and $T_{n}$ is Lipschitzian with constant $L_{n}=1+t_{n} k_{n}\left(1+s_{n} k_{n}-s_{n}\right)-t_{n} \geq 1$ for $k_{n} \geq 1$. Since $L_{n}-1=$ $t_{n}\left(1+s_{n} k_{n}\right)\left(k_{n}-1\right) \leq(1+L)\left(k_{n}-1\right)$, where $L=\sup _{n \geq 1} k_{n}$, we see that $\sum_{n}\left(L_{n}-1\right)$ converges. Now repeating the arguments in the proof of Theorem 3.1, we arrive at the following conclusions:

(i) $\lim \left\|x_{n}-f\right\|$ exists for every $f \in F(T)$.

(ii) $\left\langle p-q, J\left(f_{1}-f_{2}\right)\right\rangle=0$ for every $p, q \in \omega_{w}\left(x_{n}\right)$ and $f_{1}, f_{2} \in F(T)$.

(iii) $\lim _{n \rightarrow \infty}\left\|x_{n}-T^{n} y_{n}\right\|=0$ with $y_{n}=s_{n} T^{n} x_{n}+\left(1-s_{n}\right) x_{n}$. 
Since

$$
\begin{aligned}
\left\|T^{n} x_{n}-x_{n}\right\| & \leq\left\|T^{n} x_{n}-T^{n} y_{n}\right\|+\left\|T^{n} y_{n}-x_{n}\right\| \\
& \leq k_{n}\left\|x_{n}-y_{n}\right\|+\left\|T^{n} y_{n}-x_{n}\right\| \\
& =k_{n} s_{n}\left\|T^{n} x_{n}-x_{n}\right\|+\left\|T^{n} y_{n}-x_{n}\right\|,
\end{aligned}
$$

we have

$$
\left\|T^{n} x_{n}-x_{n}\right\| \leq \frac{1}{1-k_{n} s_{n}}\left\|T^{n} y_{n}-x_{n}\right\|,
$$

from which, together with the facts that $\left\{s_{n}\right\}$ is bounded away from 1 and $\left\{k_{n}\right\}$ converges to 1 , we conclude that $\lim _{n \rightarrow \infty}\left\|T^{n} x_{n}-x_{n}\right\|=0$. By Lemma 2.5, we have the following result:

(iv) $\lim _{n \rightarrow \infty}\left\|x_{n}-T x_{n}\right\|=0$.

It follows from (iv) and Lemma 2.6 that $\omega_{w}\left(x_{n}\right) \subset F(T)$. So to show the theorem, it suffices to show that $\omega_{w}\left(x_{n}\right)$ is a singleton. To this end, we suppose first that $X$ satisfies Opial's condition. Let $p, q$ be in $\omega_{w}\left(x_{n}\right)$ and $\left\{x_{n_{i}}\right\}$ and $\left\{x_{m_{j}}\right\}$ be subsequences of $\left\{x_{n}\right\}$ chosen so that $x_{n_{i}} \rightarrow p$ and $x_{m_{j}} \rightarrow q$. If $p \neq q$, then Opial's condition of $X$ implies that

$$
\begin{aligned}
\lim _{n \rightarrow \infty}\left\|x_{n}-p\right\| & =\lim _{i \rightarrow \infty}\left\|x_{n_{i}}-p\right\|<\lim _{i \rightarrow \infty}\left\|x_{n_{i}}-q\right\|=\lim _{j \rightarrow \infty}\left\|x_{m_{j}}-q\right\| \\
& <\lim _{j \rightarrow \infty}\left\|x_{m_{j}}-p\right\|=\lim _{n \rightarrow \infty}\left\|x_{n}-p\right\| .
\end{aligned}
$$

This contradiction proves the theorem in case $X$ satisfies Opial's condition. Next, we assume that $X$ has a Fréchet differentiable norm. Then since $\omega_{w}\left(x_{n}\right)$ $\subset F(T)$, as in the proof of Theorem 3.1, we derive from (ii) that for every $p$, $q$ in $\omega_{w}\left(x_{n}\right)$

$$
\|p-q\|^{2}=\langle p-q, J(p-q)\rangle=0 .
$$

This completes the proof.

\section{ACKNOWLEDGMENT}

The authors thank the referee for his careful reading and helpful comments on the manuscript.

\section{REFERENCES}

1. S. C. Bose, Weak convergence to the fixed point of an asymptotically nonexpansive map, Proc. Amer. Math. Soc. 68 (1978), 305-308.

2. R. E. Bruck, $A$ simple proof of the mean ergodic theorem for nonlinear contractions in Banach spaces, Isreal J. Math. 32 (1979), 107-116.

3. D. van Dulst, Equivalent norms and the fixed point property for nonexpansive mappings, $\mathrm{J}$. London Math. Soc. (2) 25 (1982), 139-144.

4. K. Goebel and W. A. Kirk, A fixed point theorem for asymptotically nonexpansive mappings, Proc. Amer. Math. Soc. 35 (1972), 171-174.

5. R. Huff, Banach spaces which are nearly uniformly convex, Rocky Mountain J. Math. 10 (1980), 743-749.

6. S. Ishikawa, Fixed points by a new iteration method, Proc. Amer. Math. Soc. 44 (1974), 147-150.

7. Z. Opial, Weak convergence of the sequence of successive approximations for nonexpansive mappings, Bull. Amer. Math. Soc. 73 (1967), 595-597. 
8. G. B. Passty, Construction of fixed points for asymptotically nonexpansive mappings, Proc. Amer. Math. Soc. 84 (1982), 213-216.

9. S. Reich, Weak convergence theorems for nonexpansive mappings in Banach spaces, J. Math. Anal. Appl. 67 (1979), 274-276.

10. J. Schu, Weak and strong convergence to fixed points of asymptotically nonexpansive mappings, Bull. Austral. Math. Soc. 43 (1991), 153-159.

11. Iterative construction of fixed points of asymptotically nonexpansive, J. Math. Anal. Appl. 158 (1991), 407-413.

12. K. K. Tan and $\mathrm{H} . \mathrm{K}$. Xu, The nonlinear ergodic theorem for asymptotically nonexpansive mappings in Banach spaces, Proc. Amer. Math. Soc. 114 (1992), 399-404.

13. _ A nonlinear ergodic theorem for asymptotically nonexpansive mappings, Bull. Austral. Math. Soc. 45 (1992), 25-36.

14. H. K. Xu, Existence and convergence for fixed points of mappings of asymptotically nonexpansive type, Nonlinear Anal. 16 (1991), 1139-1146.

Department of Mathematics, Statistics and Computing Science, Dalhousie univerSITY, Nova Scotia, CANADa B3H 3J5

E-mail address: kktan@cs.dal.ca

Institute of Applied Mathematics, East China University of Science and Technology, Shanghai 200237, China

Current address: Department of Mathematics, University of Durban-Westville, Private Bag X54001, Durban 4000, South Africa

E-mail address: hkxu@pixie.udw.ac.za 